insight into heparin conformations in solution--but so far as can be seen the obstacle of heparin heterogeneity must in every case limit the information that can be gained. Any useful procedure should include studies of heparins from different tissues and species; lung and mucosal heparins have been known for many years to differ chemically to a small but consistent extent (do the mast cells differ?) and, since this difference is now known to be paralleled by differences in relative activity at different stages in the coagulation cascade, its significance needs to be examined.

One of the driving forces behind this work has been the importance of heparin in clinical practice, including, especially, its use for antithrombotic prophylaxis following major surgery (see Heparin: Chemistry and Clinical Usage (eds Kakar and Thomas) Academic, London, 1976). It is generally agreed that in this context, where prevention of venous thrombosis must not be accompanied by a tendency to haemorrhage, antithrombin potentiation, as determined by the so-called anti-Xa assay, is as yet the best criterion of efficacy. A recent observation of Thomas and coworkers (Lancet i, 120; 1977) is therefore disturbing; it demonstrates that a semisynthetic heparin-like compound, which has negligible anti-Xa activity in vitro, nevertheless shows excellent antithrombin potentiation in the human subject. In heparin therapy the correlation of assay results in vitro with consequences in vivo has been taken on trust perhaps more than in other fields, and this will also need reconsideration.

\section{Probing facilitated transport}

\section{from S. J. Smith}

INSULIN acts on fat cell plasma membranes to promote glucose uptake and its stimulation of the utilisation of this sugar seems to be amplified by the production of a 'second messenger'. The glucose transport system in adipocytes has been well characterised as a carrier-mediated facilitated diffusion mechanism which shows bidirectionality, stereospecificity and saturation kinetics. However, little is known about the system in structural terms.

Aryl azide derivatives provide a powerful technique--photo-affinity labelling-for identifying sites of biological importance. Irradiation of the probe causes photolysis of the azido moiety producing a highly reactive nitrene, which can react covalently with any groups that lie within reach. In order to provide any interpretable information, the reagent must be shown to bind at the required locus before photolysis. Singer and coworkers have recognised the possibility of 'pseudo-affinity' labelling, which could occur, where the photolytic intermediate is able to bind reversibly to and dissociate from the active site before a covalent bond is formed. In such cases, the process would be analogous to affinity labelling. Attempts have been made to use this technique to label biological receptors in various systems.

Recently, a photoreactive aryl azide derivative of glucose, $\mathrm{N}$-(4-azido-2 nitrophenyl)-2-amino-2-deoxy-D-glucose has been used to label adipocyte membranes (Trosper \& Levy J. biol. Chem. 252, 181; 1977). In the absence of light it has been shown to inhibit the uptake of D-glucose and 3-O-methyl glucose competitively, and conversely, the uptake of the probe is decreased in the presence of glucose. Measurement of the initial uptake of the probe gave values for $K_{\mathrm{t}}$ and $V_{\max }$ similar to those obtained for glucose in identical conditions, suggesting that both sugars are being transported by the same system. When intact adipocytes were treated with the radiochemically-labelled derivative, on photolysis only four hot membrane components could be identified in SDS polyacrylamide gels of the purified plasma membrane fraction. When homogenised material was used most of the membrane proteins become labelled. Two of the specifically labelled components were found to be glycoproteins with apparent molecular weights 100,000 and 81,000 . In the presence of glucose, labelling of these components was significantly reduced, suggesting their involvement in the glucose transport system. Two lower molecular weight proteins, located on the external surface of the adipocyte membrane were also modified, but substrate protection could not be demonstrated.

The participation of membrane phosphorylation in the regulation of permeability has been implicated in, for example, toad bladder and rat brain synaptic membranes and two reports suggest that this process may be involved in modulating the effect of insulin on glucose uptake. Cuatrecasas (J. biol. Chem. 249, 3170; 1974; 249, 6854 ; 1974) reported that low concentrations of exogenous ATP can inhibit insulin-stimulated glucose transport and that this effect is associated with the phosphorylation of two membrane components of molecular weights 22,000 and 16,000 which can be separated from unlabelled components by SDS polyacrylamide gel electrophoresis. Phloretin, a strong competitive inhibitor of glucose transport, can pro- tect cells from the ATP effect with concomitant reduction in the phosphorylation of both these components. In these experiments the binding of ${ }^{123}$ I-labelled insulin was unaffected, as was the anti-lipolytic effect of the hormone. In further studies, it was found that in the presence of cyclic AMP, but not cyclic GMP, the same membrane proteins were rapidly phosphorylated, and a $\mathrm{Ca}^{2+}$-sensitive cyclicAMP dependent kinase was isolated from purified adipocyte plasma membranes. This system could not be found in guinea pig adipocytes, where glucose transport is not sensitive to insulin. The physiological importance of these findings is difficult to assess, particularly as no direct effects of insulin or adrenaline could be obtained by pretreatment of the cells with hormone before purification of the membranes.

However, more recently Avruch et al. (J. biol. Chem. 251, 1511; 1976) have shown a significant increase in the phosphorylation of a 26,000 molecular weight component of adipocyte plasma membrane, in the presence of adrenaline (even at physiological concentrations) and it seems that this effect is reversed when insulin is also present. The relation, if any, of this protein to that described by Cuatrecasas is obscure. Higher molecular weight membrane components $(79,000$ and 62,000 ) could also be phosphorylated, but the extent of this was not hormone sensitive.

The development of the aryl azide derivative of glucose, which seems to be a potential probe for the facilitated glucose transport system provides an obvious strategy for the next step, determination of whether these phosphorylated proteins are involved in the regulation of glucose uptake.

\section{Plugging the gap in the astronomical spectrum}

from a Correspondent

THE last gap in the electromagnetic spectrum available for observation by astronomers seems likely to be plugged in the near future, with the development of instruments purpose built for operating in the 1 to $10 \mathrm{~mm}$ band, between infrared and radio astronomy. One such instrument, with a dish $10 \mathrm{~m}$ across, has been proposed by observers at CalTech, where a small test dish has already been built, At the meeting of the Royal Astronomical Society on 11 March R. E. Hills, of the Mullard 